\title{
MODELAGEM DA MASSA ESPECÍFICA BÁSICA DOS CAVACOS A PARTIR DE VARIÁVEIS DENDROMÉTRICAS E DA MASSA ESPECÍFICA DO DISCO
}

\author{
Rozane Eisfeld ${ }^{1}$, Cristiane de Loyola Eisfeld ${ }^{2}$, José Mário Ferreira ${ }^{3}$, Andréia Pimentel ${ }^{4}$, \\ Fabiano Rodrigues ${ }^{5}$, Adriano Almeida ${ }^{5}$ \\ ${ }^{1}$ Enga ${ }^{\mathrm{a}}$. Florestal, M.Sc., Consultora da Silviconsult, Curitiba, PR, Brasil - rozane@ silviconsult.com.br \\ ${ }^{2}$ Eng $^{\mathrm{a}}$. Florestal, Mestranda em Ciências Florestais, UFPR, Curitiba, PR, Brasil - ceisfeld@ stcp.com.br \\ ${ }^{3}$ Eng. Florestal, M.Sc., RMS, Curitiba, PR, Brasil - jferreira@ resourcemgt.com \\ ${ }^{4}$ Eng $^{\text {a }}$. Florestal, Mestranda em Ciências Florestais, Klabin, Lages, SC, Brasil - anpimentel@ klabin.com.br \\ ${ }^{5}$ Eng. Florestal, M.Sc., International Paper, Mogi-Guaçu, SP, Brasil - fabiano.rodrigues@ipaperbr.com - adriano.almeida@ipaperbr.com
}

Recebido para publicação: 28/10/2007 - Aceito para publicação: 27/04/2009

\begin{abstract}
Resumo
O presente trabalho teve como objetivo desenvolver equações matemáticas para a estimativa da massa específica dos cavacos. Utilizaram-se dados de 560 árvores de plantios clonais de Eucalyptus sem desbaste, provenientes da empresa International Paper do Brasil. Ajustou-se um modelo a partir de variáveis dendrométricas de fácil obtenção (altura, sítio, idade e diâmetro), e outro modelo em função da idade e da massa específica do disco. As estatísticas de ajuste, incluindo a massa específica do disco, mostraram-se bem superiores, quando se consideraram apenas as variáveis dendrométricas. Observaram-se diferenças estatísticas na massa específica dos cavacos entre os quatro materiais genéticos testados, destacando os maiores valores nas unidades de manejo menos produtivas.

Palavras-chave: Massa específica; modelos de regressão; plantios clonais de Eucalyptus.
\end{abstract}

\begin{abstract}
Chip density modelling using dendrometric variables and disk density. The objective of this work was to develop mathematic equations to estimate chip density. The data came from 560 trees from Eucalyptus clonal unthinned stands owned by the International Paper of Brazil Co. It was adjusted a model using dendrometric variables easily obtained (height, site, age, and diameter) and another model using the disk density and age. The statistic of the adjustment, including the density of disk, were well above when it was considered only the dendrometric variables. It was observed statistic difference in density of chips among the four genetic materials, highlighting the highest values in less productive management units

Keywords: Density; regression models; clonal Eucalyptus plantations.
\end{abstract}

\section{INTRODUÇÃO}

A massa específica da madeira é o resultado de uma complexa combinação dos seus constituintes internos. É uma propriedade muito importante e fornece inúmeras informações sobre as características da madeira, devido a sua íntima relação com várias outras propriedades, tornando-se um parâmetro muito utilizado para qualificar a madeira, nos diversos segmentos da atividade industrial. Quase sempre, a massa específica se apresenta correlacionada com a retratibilidade, a secagem, a trabalhabilidade, a impregnabilidade, a durabilidade natural e várias propriedades mecânicas.

Para Lima et al. (2000), a massa específica aumenta muito rapidamente durante o período juvenil, cresce mais lentamente numa fase intermediária da idade, até se tornar mais ou menos constante na fase da maturidade da árvore. Segundo Tomazello Filho (1985), Silva et al. (2004) e Alzate et al. (2005), as variações da massa específica no sentido longitudinal e radial para a madeira de Eucalyptus estão relacionadas com a idade da árvore, a amostragem, o genótipo e as condições ambientais.

Estudos desenvolvidos por Trevisan et al. (2008) comprovaram a variação no sentido radial (medula/casca) da massa específica básica da madeira de Eucalyptus grandis, tendo observado menores 
valores para essa característica tecnológica na região da medula, seguidos de acréscimo até a região mais periférica do tronco.

Entre as propriedades físicas da madeira, a massa específica básica é a mais utilizada como parâmetro de seleção, sendo influenciada pela interação dos fatores de base genética com as condições ambientais (RUY, 1998). A massa especifica básica da madeira pode ser utilizada como índice seguro para avaliar o tipo de madeira produzida diante de suas correlações com as diferentes propriedades fisicomecânicas, estando, portanto, associada também às mais variadas formas de sua utilização (BRASIL et al., 1982).

O crescimento da massa específica da madeira pode ser o resultado do aumento da espessura da parede celular das fibras ou de um aumento na proporção das fibras em relação à proporção de vasos. De maneira inversa, um aumento na proporção de vasos, com ou sem decréscimo na espessura da parede celular, leva à redução na massa específica. Segundo Panshin; De Zeeuw (1980), apud Silva e Oliveira (2003), os efeitos são interativos e difíceis de ser avaliados isoladamente.

Para a indústria de celulose e papel, uma avaliação adequada da massa específica básica da madeira fornece indicações bastante precisas acerca da impregnação dos cavacos e do rendimento do processo, e geralmente está associada às características de qualidade e resistências fisicomecânicas da polpa (OLIVEIRA et al., 2004).

O objetivo principal deste estudo foi analisar as relações de variação da massa específica com o sítio, a idade e os materiais genéticos, além de ajustar modelos para a sua estimativa, utilizando dados de uma floresta de Eucalyptus sem desbaste.

\section{MÉTODOS E MATERIAIS}

\section{Localização}

A área utilizada no estudo foi de 34.540 hectares de plantio, localizada na região Sudeste do Brasil, no estado de São Paulo. A área foi dividida em três regiões, sendo Mogi-Guaçu, Brotas e São Simão os municípios correspondentes a cada uma delas. As regiões localizam-se na porção centro-leste, central e nordeste de São Paulo, conforme mostra a figura 1.

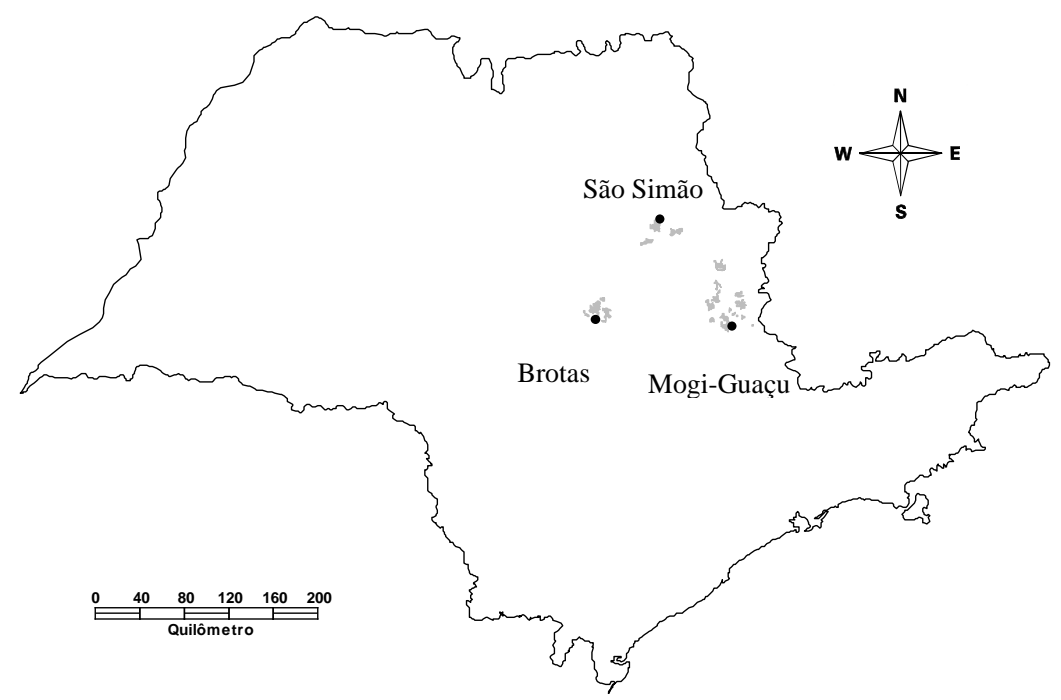

Figura 1. Posição geográfica dos municípios de Mogi-Guaçu, Brotas e São Simão e das áreas de estudo no estado de São Paulo.

Figure 1. Geographic position of Mogi-Guaçu, Brotas e São Simão cities and the studied áreas in São Paulo State.

\section{Principais características das regiões de estudo}

Região de Mogi-Guaçu: possui uma área de 17.268 hectares, num raio de $35 \mathrm{~km}$ do município de Mogi-Guaçu ( $\left.22^{\circ} 21^{\prime} S, 48^{\circ} 58^{\prime} \mathrm{O}\right)$. O clima da região é do tipo Cwa, segundo a classificação de Koeppen, 
com temperatura anual média de $21{ }^{\circ} \mathrm{C}$, pluviosidade média anual variando entre 900 e $1.900 \mathrm{~mm}$, concentrando-se entre os meses de outubro e março, e déficit hídrico de $36 \mathrm{~mm}$. Os solos predominantes na região são os Latossolos Vermelho-Amarelos, Latossolos Vermelhos e Argissolos (MORAES et al., 1993a, 1993b, 1993c, 1993c, 1994a, 1994b, 1995d, 1995e; DEMATTÊ, 2000).

Região de Brotas: possui uma área de 8.838 hectares, num raio de $10 \mathrm{~km}$ do município de Brotas $\left(22^{\circ} 13^{\prime} \mathrm{S}, 48^{\circ} 01^{\prime} \mathrm{O}\right)$. O clima da região é do tipo Cwa, segundo a classificação de Köeppen, com temperatura anual média de $19{ }^{\circ} \mathrm{C}$, pluviosidade média anual variando entre 1.100 e $2.400 \mathrm{~mm}$, concentrando-se entre os meses de outubro a abril, e déficit hídrico de $63 \mathrm{~mm}$. Os solos predominantes na região são os Latossolos Vermelho-Amarelos e os Neossolos Quartzarênicos (MORAES et al., 1993c, 1993e; DEMATTÊ, 2000).

Região de São Simão: possui uma área de 8.434 hectares, num raio de $30 \mathrm{~km}$ do município de São Simão $\left(22^{\circ} 29^{\prime} S, 47^{\circ} 34^{\prime}\right.$ O). O clima da região é do tipo Cwa, segundo a classificação de Köeppen, com temperatura anual média de $22{ }^{\circ} \mathrm{C}$, pluviosidade média anual variando entre 1.100 e $1.700 \mathrm{~mm}$, concentrando-se entre os meses de outubro e março, e déficit hídrico de $110 \mathrm{~mm}$. Os solos predominantes na região são os Neossolos Quartzarênicos (MORAES et al., 1994f, 1994g; DEMATTÊ, 2000).

\section{Material genético}

Foram avaliadas diferentes plantações de clones de Eucalyptus urophylla e Eucalyptus grandis, previamente selecionadas em testes clonais específicos e aprovados em plantios comerciais extensos. Essas plantações estão identificadas neste estudo como clones A, B, C, D.

\section{Unidades de manejo - sítio}

Todos os talhões da empresa estão agrupados em oito diferentes unidades de manejo, que foram definidas considerando-se as características de solo que mais interferem na produtividade da floresta. Nas unidades de manejo 1 e 2 estão localizados os sítios de melhor produtividade; nas unidades 7 e 8 estão os de pior produtividade.

\section{Metodologia de campo}

Foram amostradas 560 árvores, distribuídas nos quatro materiais genéticos, idades e unidades de manejo. A combinação entre os materiais, com 3 classes de idade e 3 grupos de unidades de manejo, definiu 36 estratos diferentes, servindo de base para a distribuição das árvores amostradas e para a obtenção do rendimento.

Primeiramente, foram definidos os talhões que abrangessem uniformemente os diferentes materiais, unidades e idades. Em cada um deles foram selecionadas duas parcelas. Em cada parcela, a amostragem ocorreu em, no máximo, 4 árvores. As árvores que foram cortadas estavam plantadas próximas à borda da parcela.

Em cada árvore foram coletados 5 toretes de $50 \mathrm{~cm}$ de comprimento, retirados na base, a 25\%, a $50 \%$, a $75 \%$ e a $100 \%$ da altura comercial. Além disso, houve a coleta de dois discos no DAP para a determinação da massa específica do disco. Nas fichas de campo, anotaram-se a localização, o diâmetro, a altura total e a comercial de cada árvore e, também, a data da coleta. Os toretes e os discos foram levados ao laboratório de química da madeira na própria empresa, onde se realizou a determinação da massa específica do cavaco e do disco.

\section{Metodologia laboratorial}

A massa específica é a relação da massa seca com o volume saturado, como segue:

$$
M e_{\text {Básica }}=\frac{\text { Peso }_{\text {Seco }}}{\text { Volume }_{\text {Saturado }}}
$$

Em que: $\mathrm{Me}=$ massa específica $\mathrm{g} / \mathrm{cm}^{3}$.

Para que fosse possível a determinação da massa seca primeiramente dos discos, alguns procedimentos laboratoriais foram seguidos. Os discos foram colocados em sacos plásticos perfurados, imersos em tambores com água e submetidos a vácuo para eliminar todo o ar contido nos discos. Após 
completa saturação, obteve-se o volume em uma balança hidrostática, através do método de deslocamento. Para a massa seca, as amostras foram colocadas em estufa com temperatura de $103 \pm 2{ }^{\circ} \mathrm{C}$ por, aproximadamente, 36 horas. Feito isso, realizou-se a pesagem, obtendo-se a massa seca.

Para a determinação da massa seca, os cavacos foram selecionados e colocados em um recipiente com capacidade de um litro. Em seguida, foram acondicionados em sacos plásticos perfurados e imersos em tambores. Depois de saturados, foram submetidos a vácuo, de forma semelhante à utilizada para os discos. Os cavacos, então, foram colocados em cestos e assim foi obtido o volume saturado pelo método de deslocamento. Para a massa seca, os cavacos foram levados à estufa em becker com temperatura de $103 \pm 2{ }^{\circ} \mathrm{C}$, por 36 horas. Posteriormente realizou-se a pesagem e se obteve a massa seca.

\section{Metodologia final - escritório}

Para a determinação da massa específica dos cavacos, realizou-se um estudo prévio das variáveis independentes, buscando-se conhecer o comportamento dos dados e a influência na massa específica, através de análises estatísticas.

Para cada material genético, foram testadas duas equações, uma não incluindo a massa específica do disco como variável independente. Ambas foram ajustadas pelo método de escolha de variáveis forward stepwise, a partir do software Statistica.

O procedimento de modelagem de regressão forward stepwise parte da variável independente mais correlacionada com a variável dependente (massa específica dos cavacos). No passo seguinte, inclui mais uma variável, que apresenta a maior correlação parcial em função do teste F (SCHNEIDER, 1998).

Para ser ajustado no procedimento forward, os modelos máximos de regressão foram: DAPxHT).

Sem disco: D = f (DAP, HT, HC, U, I, DAP ${ }^{2}, \operatorname{lnDAP}, 1 / \mathrm{DAP}, \mathrm{HT}^{2}, \operatorname{lnHT}, 1 / \mathrm{HTxDAP}^{2} \mathrm{xHT}$,

Com disco: $\mathrm{D}=\mathrm{f}\left(\mathrm{DAP}, \mathrm{HT}, \mathrm{HC}, \mathrm{U}, \mathrm{I}, \mathrm{DAP}^{2}\right.$, lnDAP, 1/DAP, HT ${ }^{2}, \operatorname{lnHT}, 1 / \mathrm{HTxDAP}^{2} \mathrm{xHT}$, DAPxHT e massa específica do disco).

Em que: $\mathrm{D}=$ massa específica dos cavacos $\left(\mathrm{kg} / \mathrm{m}^{3}\right)$;

$\mathrm{DAP}=$ diâmetro à altura do peito $(\mathrm{cm})$;

$\mathrm{HT}=$ altura total $(\mathrm{m})$;

$\mathrm{HC}=$ altura comercial $(\mathrm{m})$;

$\mathrm{I}=$ idade $(\operatorname{anos})$;

$\mathrm{U}=$ unidade de manejo.

Para testar a qualidade dos ajustes dos modelos, utilizaram-se três parâmetros de comparação: o coeficiente de determinação $\left(\mathrm{R}^{2}\right)$, o erro padrão da estimativa (Syx) e a análise gráfica dos resíduos.

\section{RESULTADOS}

Análise preliminar da relação entre a massa específica dos cavacos e as demais variáveis coletadas

Calculou-se uma matriz de correlação para verificar a relação entre a massa específica dos cavacos e as demais variáveis deste estudo, conforme a tabela 1.

Tabela 1. Matriz de correlação.

Table 1. Correlation matrix.

\begin{tabular}{lcccccc}
\hline Variável & Idade & DAP & $\begin{array}{c}\text { Altura } \\
\text { total }\end{array}$ & $\begin{array}{c}\text { Altura } \\
\text { comercial }\end{array}$ & $\begin{array}{c}\text { Dens. disco } \\
\text { Unidade de } \\
\text { manejo }\end{array}$ \\
\hline Massa específica dos cavacos & 0,56 & 0,36 & 0,39 & 0,42 & 0,89 & 0,28 \\
\hline
\end{tabular}

A matriz mostra que todos os fatores testados possuem correlação significativa $(\alpha=0,05)$ com a massa específica dos cavacos. A massa específica do disco é a variável mais fortemente correlacionada com a massa específica dos cavacos.

As análises estatísticas para cada material genético mostram que, em média, o material genético A possui a menor massa específica, e o material D possui a maior massa específica (Tabela 2). Em média, todas as 560 árvores analisadas apresentaram a massa específica de $478,65 \mathrm{~kg} / \mathrm{m}^{3}$. 
Tabela 2. Estatísticas básicas da massa específica dos cavacos por material genético.

Table 2. Basic statistics of chip density by genetic material.

\begin{tabular}{lccc}
\hline Material genético & Média $\left(\mathbf{k g} / \mathbf{m}^{\mathbf{3}}\right)$ & $\mathbf{N}$ & Desvio padrão \\
\hline $\mathrm{A}$ & 468,4405 & 252 & 27,74558 \\
$\mathrm{~B}$ & 481,7582 & 153 & 26,28363 \\
$\mathrm{C}$ & 484,6263 & 99 & 20,54293 \\
$\mathrm{D}$ & 505,5357 & 56 & 41,71983 \\
Todos os grupos & 478,6500 & 560 & 30,11835 \\
\hline
\end{tabular}

Tabela 3. Análise de variância da massa específica dos cavacos por material genético.

Table 3. Variance analysis for chip density by genetic material.

\begin{tabular}{lcccccccc}
\hline Variável & SS & Df & MS & SS & df & MS & F & P \\
\hline Massa específica dos cavacos & 71760,14 & 3 & 23920,05 & 435317,3 & 556 & 782,9447 & 30,55139 & 0,000000 \\
\hline
\end{tabular}

A análise de variância mostra que há diferença significativa entre as médias por material genético $(\alpha=0,05)$.

Tabela 4. Teste de média (Tukey) da massa específica dos cavacos por material genético.

Table 4. Average test (Tukey) for chip density by genetic material.

\begin{tabular}{ccccc}
\hline & $\mathbf{A}$ & $\mathbf{B}$ & $\mathbf{C}$ & $\mathbf{D}$ \\
\hline $\mathrm{A}$ & & 0,000027 & 0,000014 & 0,000008 \\
$\mathrm{~B}$ & 0,000027 & & 0,856899 & 0,000008 \\
$\mathrm{C}$ & 0,000014 & 0,856899 & & 0,000053 \\
D & 0,000008 & 0,000008 & 0,000053 & \\
\hline
\end{tabular}

Apenas as médias dos materiais $\mathrm{B}$ e $\mathrm{C}$ não diferem estatisticamente entre si, segundo o teste de Tukey em nível de 5\% de probabilidade. Portanto, para uma estimativa mais precisa, faz-se necessário o ajuste da massa específica dos cavacos por material genético.

\section{Resultados para o material genético "A"}

Para o material genético A, as formulações matemáticas resultantes do ajuste pelo método forward stepwise foram:

a) Sem disco: $D=401,6726+17,3561 \times I+3,1218 \times U-1,4242 \times H T$

a) Com disco: $D=111,3681+0,7078 \times$ disco $+3,9671 \times I$

Em que: $D=$ massa específica dos cavacos $\left(\mathrm{kg} / \mathrm{m}^{3}\right)$;

$I=$ idade (anos);

$U=$ unidade de manejo;

$H T=$ altura total $(\mathrm{m})$;

disco $=$ massa específica do disco $\left(\mathrm{kg} / \mathrm{m}^{3}\right)$.

Table 5. Regression statistics for both presented equations.

\begin{tabular}{lcccc}
\hline Modelos & $\mathbf{t}$ & $\mathbf{S y x}\left(\mathbf{k g} / \mathbf{m}^{\mathbf{3}}\right)$ & $\mathbf{S y x} \mathbf{( \% )}$ & $\mathbf{R}^{\mathbf{2}}$ \\
\hline Sem disco & 248 & 17,96 & 3,83 & $58,07 \%$ \\
Com disco & 249 & 11,43 & 2,44 & $83,00 \%$ \\
\hline
\end{tabular}

O segundo ajuste, que inclui a massa específica de disco como uma variável independente, apresentou estatística de ajuste superior ao modelo que não inclui o disco, com coeficiente de determinação de $83 \%$, erro padrão da estimativa de apenas $2,44 \%\left(11 \mathrm{~kg} / \mathrm{m}^{3}\right)$ e resíduos sem tendenciosidades.

Vale a pena ressaltar, no entanto, que a variável massa específica do disco é difícil de ser obtida, requerendo o corte de árvore, a retirada do disco e a sua análise no laboratório. Em função disso, o primeiro ajuste passa a ser economicamente mais vantajoso, pois a massa específica é estimada apenas em função de variáveis facilmente obtidas pelo inventário florestal. Os resultados desse ajuste estão apresentados na tabela 6 . 
Tabela 6. Tabela de massa específica por idade e unidade de manejo (ajuste sem massa específica do disco).

Table 6. Density table by age and management unit (adjustment without disk density).

\begin{tabular}{lccccccccc}
\hline Idade & Altura & $\mathbf{1}$ & $\mathbf{2}$ & $\mathbf{3}$ & $\mathbf{4}$ & $\mathbf{5}$ & $\mathbf{6}$ & $\mathbf{7}$ & $\mathbf{8}$ \\
\hline 2 & 12,8 & 421 & 424 & 427 & 431 & 434 & 437 & 440 & 443 \\
3 & 15,8 & 434 & 437 & 441 & 444 & 447 & 450 & 453 & 456 \\
4 & 20,6 & 445 & 448 & 451 & 454 & 457 & 461 & 464 & 467 \\
5 & 22,7 & 459 & 462 & 466 & 469 & 472 & 475 & 478 & 481 \\
6 & 26,0 & 472 & 475 & 478 & 481 & 484 & 488 & 491 & 494 \\
7 & 28,6 & 486 & 489 & 492 & 495 & 498 & 501 & 504 & 507 \\
8 & 31,8 & 498 & 501 & 505 & 508 & 511 & 514 & 517 & 520 \\
\hline
\end{tabular}

\section{Resultados para o material genético "B"}

Para o material genético $\mathrm{B}$, as formulações matemáticas resultantes do ajuste pelo método forward stepwise foram:

b) Sem disco: $D=417,2158+20,1851 \times I+3,3100 \times U-0,0951 \times H T^{2}$

c) Com disco: $D=115,6227+0,7175 \times$ disco $+3,5268 \times I$

Em que: $D=$ massa específica dos cavacos $\left(\mathrm{kg} / \mathrm{m}^{3}\right)$;

$$
\begin{aligned}
& I=\text { idade }(\text { anos }) ; \\
& U=\text { unidade de manejo; } \\
& H T=\text { altura total }(\mathrm{m}) ; \\
& \text { disco = massa específica do disco }\left(\mathrm{kg} / \mathrm{m}^{3}\right) .
\end{aligned}
$$

Nos dois materiais genéticos (A e B) foram selecionadas as mesmas variáveis para a estimativa da massa específica.

Tabela 7. Estatísticas de ajuste para as duas equações apresentadas.

Table 7. Regression statistics for both presented equations.

\begin{tabular}{lcccc}
\hline Modelos & $\mathbf{t}$ & $\mathbf{S y x}\left(\mathbf{k g} / \mathbf{m}^{\mathbf{3}}\right)$ & $\mathbf{S y x}(\boldsymbol{\%})$ & $\mathbf{R}^{\mathbf{2}}$ \\
\hline Sem disco & 149 & 19,32 & 4,01 & $45,94 \%$ \\
Com disco & 150 & 11,58 & 2,40 & $80,57 \%$ \\
\hline
\end{tabular}

O segundo ajuste, que inclui a massa específica de disco como uma variável independente, apresentou estatística de ajuste superior ao modelo que não inclui o disco, com coeficiente de determinação de $81 \%$, erro padrão da estimativa de $2,40 \%\left(11,6 \mathrm{~kg} / \mathrm{m}^{3}\right)$ e resíduos sem tendenciosidades.

Tabela 8. Tabela de massa específica por idade e unidade de manejo (ajuste sem massa específica do disco).

Table 8. Density table by age and management unit (adjustment without disk density).

\begin{tabular}{lccccccccc}
\hline Idade & Altura & $\mathbf{1}$ & $\mathbf{2}$ & $\mathbf{3}$ & $\mathbf{4}$ & $\mathbf{5}$ & $\mathbf{6}$ & $\mathbf{7}$ & $\mathbf{8}$ \\
\hline 2 & 12,8 & 445 & 449 & 452 & 455 & 458 & 462 & 465 & 468 \\
3 & 15,8 & 457 & 461 & 464 & 467 & 470 & 474 & 477 & 480 \\
4 & 20,6 & 461 & 464 & 468 & 471 & 474 & 478 & 481 & 484 \\
5 & 22,7 & 473 & 476 & 479 & 483 & 486 & 489 & 492 & 496 \\
6 & 26,0 & 478 & 481 & 484 & 487 & 491 & 494 & 497 & 501 \\
7 & 28,6 & 484 & 487 & 491 & 494 & 497 & 501 & 504 & 507 \\
8 & 31,8 & 486 & 489 & 492 & 496 & 499 & 502 & 505 & 509 \\
\hline
\end{tabular}

\section{Resultados para o material genético "C"}

Para o material genético $\mathrm{C}$, as formulações matemáticas resultantes do ajuste pelo método forward stepwise foram: 
a) Sem disco: $D=385,2143+15,2484 \times I+0,8269 \times U+697,5454 \times \frac{1}{H T}$

b) Com disco: $D=192,432+0,6340 \times$ disco $-188,002 \times \frac{1}{D A P}$

Em que: $D=$ massa específica dos cavacos $\left(\mathrm{kg} / \mathrm{m}^{3}\right)$;

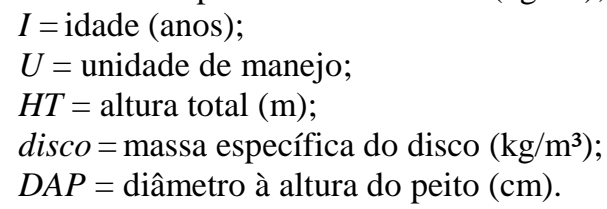

Tabela 9. Estatísticas de ajuste para as duas equações apresentadas.

Table 9. Regression statistics for both presented equations.

\begin{tabular}{lcccc}
\hline Modelos & $\mathbf{t}$ & Syx $\left(\mathbf{k g} / \mathbf{m}^{\mathbf{3}}\right)$ & $\mathbf{S y x}(\boldsymbol{\%})$ & $\mathbf{R}^{\mathbf{2}}$ \\
\hline Sem disco & 95 & 18,27 & 3,76 & $20,86 \%$ \\
Com disco & 96 & 9,31 & 1,92 & $79,46 \%$ \\
\hline
\end{tabular}

O segundo ajuste, que inclui a massa específica de disco como uma variável independente, apresentou estatística de ajuste bem superior ao modelo que não inclui o disco, com coeficiente de determinação de $79 \%$, erro padrão da estimativa de $1,92 \%\left(9,3 \mathrm{~kg} / \mathrm{m}^{3}\right)$ e resíduos sem tendenciosidades.

O coeficiente de determinação do modelo sem disco obtido para o material C $(21 \%)$ foi inferior aos obtidos nos demais (58\% no material A e $46 \%$ no material B). Esse material entrou no plantio comercial da empresa no ano de 2002, portanto sua amostragem ficou restrita a talhões com menos de cinco anos e distribuídos apenas nas unidades de manejo 2, 3, 4, 5 e 6. Possivelmente, a falta de dados nas idades mais avançadas e nos pontos extremos das unidades de manejo (1, 7 e 8$)$ pode ter afetado o nível de acuracidade do ajuste.

Tabela 10 Tabela de massa específica por idade e unidade de manejo (ajuste sem massa específica do disco).

Table 10. Density table by age and management unit (adjustment without disk density).

\begin{tabular}{lccccccccc}
\hline Idade & Altura & $\mathbf{1}$ & $\mathbf{2}$ & $\mathbf{3}$ & $\mathbf{4}$ & $\mathbf{5}$ & $\mathbf{6}$ & $\mathbf{7}$ & $\mathbf{8}$ \\
\hline 2 & 12,8 & 471 & 472 & 472 & 473 & 474 & 475 & 476 & 477 \\
3 & 15,8 & 476 & 477 & 477 & 478 & 479 & 480 & 481 & 482 \\
4 & 20,6 & 481 & 482 & 483 & 483 & 484 & 485 & 486 & 487 \\
5 & 22,7 & 493 & 494 & 495 & 496 & 496 & 497 & 498 & 499 \\
6 & 26,0 & 504 & 505 & 506 & 507 & 508 & 509 & 509 & 510 \\
7 & 28,6 & 517 & 518 & 519 & 520 & 520 & 521 & 522 & 523 \\
8 & 31,8 & 530 & 531 & 532 & 532 & 533 & 534 & 535 & 536 \\
\hline
\end{tabular}

\section{Resultados para o material genético " $D$ "}

Para o material genético D, as formulações matemáticas resultantes do ajuste pelo método forward stepwise foram:

a) Sem disco: $D=386,8904+27,6424 \times I+4,8855 \times U-0,0842 \times H^{2}$

b) Com disco: $D=104,4750+0,7098 \times$ disco $+7,1542 \times I$

Em que: $D=$ massa específica dos cavacos $\left(\mathrm{kg} / \mathrm{m}^{3}\right)$;

$I=$ idade (anos);

$U=$ unidade de manejo;

$H T=$ altura total $(\mathrm{m})$;

disco $=$ massa específica do disco $\left(\mathrm{kg} / \mathrm{m}^{3}\right)$.

Nos materiais genéticos A, B e D foram selecionadas as mesmas variáveis para a estimativa da massa específica.

FLORESTA, Curitiba, PR, v. 39, n. 4, p. 877-886, out./dez. 2009. 
Tabela 11. Estatísticas de ajuste para as duas equações apresentadas.

Table 11. Regression statistics for both presented equations.

\begin{tabular}{lcccc}
\hline Modelos & $\mathbf{t}$ & $\mathbf{S y x}\left(\mathbf{k g} / \mathbf{m}^{\mathbf{3}}\right)$ & $\mathbf{S y x} \mathbf{( \% )}$ & $\mathbf{R}^{\mathbf{2}}$ \\
\hline Sem disco & 52 & 21,55 & 4,26 & $73,30 \%$ \\
Com disco & 53 & 13,93 & 2,75 & $88,85 \%$ \\
\hline
\end{tabular}

O segundo ajuste, que inclui a massa específica de disco como uma variável independente, apresentou estatística de ajuste superior ao modelo que não inclui o disco, com coeficiente de determinação de $89 \%$, erro padrão da estimativa de $2,75 \%\left(13,9 \mathrm{~kg} / \mathrm{m}^{3}\right)$ e resíduos sem tendenciosidades.

Tabela 12. Tabela de massa específica por idade e unidade de manejo (ajuste sem massa específica do disco).

Table 12. Density table by age and management unit (adjustment without disk density).

\begin{tabular}{lccccccccc}
\hline Idade & Altura & $\mathbf{1}$ & $\mathbf{2}$ & $\mathbf{3}$ & $\mathbf{4}$ & $\mathbf{5}$ & $\mathbf{6}$ & $\mathbf{7}$ & $\mathbf{8}$ \\
\hline 2 & 12,8 & 433 & 438 & 443 & 448 & 453 & 458 & 462 & 467 \\
3 & 15,8 & 454 & 458 & 463 & 468 & 473 & 478 & 483 & 488 \\
4 & 20,6 & 467 & 472 & 477 & 481 & 486 & 491 & 496 & 501 \\
5 & 22,7 & 487 & 492 & 497 & 501 & 506 & 511 & 516 & 521 \\
6 & 26,0 & 501 & 506 & 511 & 516 & 520 & 525 & 530 & 535 \\
7 & 28,6 & 516 & 521 & 526 & 531 & 536 & 541 & 546 & 551 \\
8 & 31,8 & 528 & 532 & 537 & 542 & 547 & 552 & 557 & 562 \\
\hline
\end{tabular}

\section{Resultados com a base completa de dados}

Com toda a base de dados, as formulações matemáticas resultantes do ajuste pelo método forward stepwise foram:

a) Sem disco: $D=424,6182+23,0611 \times I+2,4660 \times U-6,4199 \times H T+5,2288 \times D A P$

b) Com disco: $D=90,81468+0,77514 \times$ disco $+2,69456 \times I$

Em que: $D=$ massa específica dos cavacos $\left(\mathrm{kg} / \mathrm{m}^{3}\right)$;

$I=$ idade (anos);

$U=$ unidade de manejo;

$H T=$ altura total $(\mathrm{m})$;

disco $=$ massa específica do disco $\left(\mathrm{kg} / \mathrm{m}^{3}\right)$;

$D A P=$ diâmetro à altura do peito $(\mathrm{cm})$.

Tabela 13. Estatísticas de ajuste para as duas equações apresentadas.

Table 13. Regression statistics for both presented equations.

\begin{tabular}{lcccc}
\hline Modelos & $\mathbf{t}$ & $\mathbf{S y x}\left(\mathbf{k g} / \mathbf{m}^{\mathbf{3}}\right)$ & Syx (\%) & $\mathbf{R}^{\mathbf{2}}$ \\
\hline Sem disco & 555 & 22,29 & 4,66 & $45,21 \%$ \\
Com disco & 557 & 12,99 & 2,71 & $81,40 \%$ \\
\hline
\end{tabular}

O segundo ajuste, que inclui a massa específica de disco como uma variável independente, apresentou estatística de ajuste superior ao modelo que não inclui o disco, com coeficiente de determinação de $81 \%$, erro padrão da estimativa de $2,71 \%\left(13 \mathrm{~kg} / \mathrm{m}^{3}\right)$ e resíduos sem tendenciosidades.

Todos os materiais testados apresentaram correlação positiva com a unidade de manejo e com a idade, mostrando que a massa específica tende a ser maior nos piores sítios.

A massa específica da madeira é uma propriedade resultante de fatores como dimensões das células, espessura, composição química da parede celular e percentuais de ocupação dos variados tipos de células. Segundo Foelkel et al. (1983), as dimensões dos elementos celulares variam em função da idade do vegetal. Com base nesses fatos, é provável que a tendência de variação da massa específica encontrada neste estudo seja alterada com o desenvolvimento e maturação da árvore.

A qualidade da madeira é afetada pela idade da árvore. É necessário definir a época de corte, não apenas em função de critérios que avaliam a produção de volume ou de matéria seca lenhosa, mas, 
também, mediante uma análise da heterogeneidade dos parâmetros que expressam a qualidade (LIMA et al., 1992). Assim sendo, os resultados deste estudo servem como subsídio para que o gestor florestal possa definir o regime de manejo que otimize a combinação entre a produção da floresta (volume) e a sua qualidade (massa específica).

Tabela 14. Tabela de massa específica por idade e unidade de manejo (ajuste sem massa específica do disco).

Table 14. Density table by age and management unit (adjustment without disk density).

\begin{tabular}{lccccccccc}
\hline Idade & Altura & $\mathbf{1}$ & $\mathbf{2}$ & $\mathbf{3}$ & $\mathbf{4}$ & $\mathbf{5}$ & $\mathbf{6}$ & $\mathbf{7}$ & $\mathbf{8}$ \\
\hline 2 & 12,8 & 444 & 446 & 448 & 451 & 453 & 456 & 458 & 461 \\
3 & 15,8 & 454 & 457 & 459 & 462 & 464 & 466 & 469 & 471 \\
4 & 20,6 & 460 & 463 & 465 & 468 & 470 & 473 & 475 & 478 \\
5 & 22,7 & 475 & 477 & 479 & 482 & 484 & 487 & 489 & 492 \\
6 & 26,0 & 482 & 484 & 487 & 489 & 492 & 494 & 497 & 499 \\
7 & 28,6 & 497 & 499 & 502 & 504 & 506 & 509 & 511 & 514 \\
8 & 31,8 & 503 & 506 & 508 & 511 & 513 & 515 & 518 & 520 \\
\hline
\end{tabular}

\section{CONCLUSÕES}

- A massa específica variou com o sítio (unidades de manejo), idade e material genético.

- A massa específica aumentou com a idade e nas unidades menos produtivas.

- O material genético D se apresentou como o clone mais denso dos estudados neste trabalho.

- A massa específica do disco obteve uma alta correlação com a do cavaco e a sua inclusão nos ajustes melhorou muito as estatísticas de regressão.

\section{RECOMENDAÇÕES}

- Utilizar, sempre que possível, a massa específica do disco para estimar a do cavaco.

- Complementar este trabalho com a amostragem de novas árvores, principalmente em talhões mais velhos plantados com o material $\mathrm{C}$.

\section{REFERÊNCIAS}

ALZATE, S. B. A.; TOMAZELLO FILHO, M.; PIEDADE, S. M. S. Variação longitudinal da densidade básica da madeira de clones de Eucalyptus grandis Hill ex Maiden, E. saligna Sm. e E. grandis x urophylla. Scientia Forestalis, Piracicaba, n. 68, p. 87-95, 2005.

BARRICHELO, L. E. G.; BRITO, J. O.; MIGLIORINI, A. J. Estudo da variação da massa específica básica em Eucalyptus spp. In: CONGRESSO FLORESTAL BRASILEIRO, 4., 1982, Belo Horizonte,. Anais... São Paulo, SBS, 1983, p. 726-731.

BRASIL, M. A. M.; MONTAGNA, R. G.; COELHO, L. C. C.; VEIGA, R. A. A. Massa específica básica da madeira de Pinus elliottii var. elliottii em três regiões do Estado de São Paulo. Boletim Técnico Instituto Florestal, v. 36, n. 1, p. 9-17, 1982.

BRASIL, M. A. M.; VEIGA, R. A. A.; FERREIRA, M. Variação da massa específica básica da madeira nas secções transversais do caule da base do tronco para a copa de eucalipto. IPEF, Piracicaba, n. 15, p. 73-82, dez., 1977.

DEMATTE, J. L. I. Solos. In: SALATI, E.; ABSY, M. L.; VICTORIA, R. L. (Eds.). Amazônia: um ecossistema em transformação. Manaus: INPA, 2000. p. 119 -162.

FOELKEL, C. E. B.; BUSNARDO, C. A.; DIAS, C.; SCHIMIDT, C.; SILVA, S. M.; VESZ, J. B. Variabilidade radial da madeira de Eucalyptus saligna. In: CONGRESSO FLORESTAL BRASILEIRO, 4, Belo Horizonte, 1982. Anais... São Paulo, SBS, 1983. p. 782-791. 
LIMA, J. T.; ROSADO, S. C. S.; OLIVEIRA, A. D. Variação da massa específica da madeira de Eucalyptus grandis, Eucalyptus tereticornis e Eucalyptus camaldulensis no sentido longitudinal dos caules. Lavras: Revista Ciência e Prática, Lavras, v. 16, n. 1, p. 123-127, 1992.

LIMA, J. T.; BREESE, M. C.; CAHALAN, C. M. Variation in wood density and mechanical properties in Eucalyptus clones. In: THE FUTURE OF EUCALYPTS FOR WOOD PRODUCTS. 2000, Launceston, Tasmania. Proceedings... Launceston: IUFRO. 2000, p. 282-291.

OLIVEIRA, R. C.; COLODETTE, J. L.; GOMIDE, J. L.; QUEIROZ, S. C. S.; Influência da massa específica básica da madeira na qualidade da polpa kraft de clones hibrídos de Eucalyptus grandis W. Hill ex Maiden X Eucalyptus urophylla S. T. Blake. In: Revista Arvore, Viçosa, MG, v. 28, n. 6, nov./dez. 2004.

PANSHIN, A. J.; ZEEUW, C. de Text book of wood technology. 4. ed. New York: Mc Graw Hill, 1980. $722 \mathrm{p}$.

RUY, O. F. Variação da qualidade da madeira em clones de Eucalyptus urophylla S. T. Blake da Ilha de Flores, Indonésia. 69 p. Dissertação (Mestrado) - Escola Superior de Agricultura "Luiz de Queiroz", Universidade de São Paulo, Piracicaba, 1998.

SCHNEIDER, P. R. Análise de regressão aplicada à engenharia florestal. 2. ed. Santa Maria: UFSM/CEPEF, 1998, $236 \mathrm{p}$.

SILVA, J. C.; OLIVEIRA, J. T. S. Variação radial da retratibilidade e massa específica básica da madeira de Eucalyptus saligna Sm. Revista Árvore, Viçosa, MG, v. 27, n. 3, p. 381-385, 2003.

SILVA, J. C.; OLIVEIRA, J. T. S.; TOMAZELLO FILHO, M.; KEINERT JÚNIOR, S.; MATOS, J. L. $\mathrm{M}$ de. Influência da idade e da posição radial na massa específica básica da madeira de Eucalyptus grandis Hill ex. Maiden. Floresta, Curitiba, v. 34, n. 1, p. 13-22, 2004.

TREVISAN, R.; HASELEIN, C. R.; MELO, R. R.; STANGERLIN, D. M.; BELTRAME, R.; GATTO, D. A.; CALEGARI, L. Variação radial da massa específica básica da madeira de Eucalyptus grandis W. Hill ex Maiden. Floresta, Curitiba, v. 38, n. 3, p.553-559, 2008.

TOMAZELLO FILHO, M. Variação radial da densidade básica e da estrutura anatômica da madeira de Eucalyptus saligna e Eucalyptus grandis. IPEF, Piracicaba, v. 29, p. 37-45, 1985. 